\title{
PROSPECTIVE OBSERVATIONAL STUDY ON MYOCARDIAL INFARCTION IN RELATIONSHIP WITH VARIOUS RISK FACTORS
}

\author{
LINU P BABY, NEEMA JOHNSON, SHYAMILY JOHN, KAMESWARAN R*, SAMBATHKUMAR R \\ Department of Pharmacy Practice, J.K.K. Nattraja College of Pharmacy, Komarapalayam, Tamil Nadu, India. \\ Email: kameswaran.r@jkkn.org
}

Received: 07 June 2016, Revised and Accepted: 31 August 2016

\section{ABSTRACT}

Objective: The aim of this study was to determine the prevalence of risk factors which influence the increased rate of myocardial infarction in a tertiary care hospital.

Methods: Data for this 6 months prospective observational study were collected from 227 patients admitted to a tertiary care hospital with acute myocardial infarction, through patient interviews and case reports and were analyzed.

Results: A total of 227 cases were taken for this study. Out of 227 patients, 168 (74.19\%) were males and 59 (25.81\%) were females. This study reveals some major risk factors which contribute the increased rate of myocardial infarction and were smoking, chronic alcoholism, obesity, sedentary lifestyle, male sex, physical inactivity, comorbid diseases, sleep disorders as well as age group between 41 and 60 years. No association between oil consumption coffee intake and a positive association between tea intake was observed. Out of 105 patients diagnosed with ST elevated MI, 55 (52.23\%) were treated with fibrinolytic therapy and 50 (47.77\%) were excluded from this therapy mainly due to delayed admission. All the patients were found to be benefited by beta-blocker therapy.

Conclusion: Results suggested that awareness regarding moderate physical activity, cessation of cigarette smoking, increased daily consumption of fruits and vegetables may reduce the increased myocardial infarction risk.

Keywords: Myocardial infarction, Risk factors, Fibrinolytic therapy, Beta blockers.

(C) 2016 The Authors. Published by Innovare Academic Sciences Pvt Ltd. This is an open access article under the CC BY license (http://creativecommons. org/licenses/by/4. 0/) DOI: http://dx.doi.org/10.22159/ajpcr.2016.v9s3.13735

\section{INTRODUCTION}

Cardiovascular disease (CVD) is the leading cause of death and disability-adjusted life years worldwide, with increasing incidence and prevalence in low- and middle-income countries (LMICs) [1]. The burden is greater in LMICs because of their larger population sizes and widespread exposure to increasing levels of risk factors such as unhealthy diet, obesity, physical inactivity, chronic alcoholism, diabetes, tobacco use, hypertension, and abnormal blood lipid profile. It has been shown that risk factors for CVD are largely similar in highincome countries as in LMICs. The consequences of globalization and urbanization are also contributory factors [2]. By 2020, more than $80 \%$ of global CV incidents will be in these countries, with the largest burden occurring in the two Asian countries, China and India, as they rapidly urbanize [3]. In India among adults over 20 years of age, the estimated prevalence of heart disease is around 3-4\% in rural areas and $8-10 \%$ in urban areas, representing a 3 -fold rise in rural areas and a 6-fold rise in urban areas between the years 1960 and 2000 [3]. Several factors such as genetic, metabolic, conventional, and non-conventional risk factors were suspected to cause high CV morbidity and mortality rates among the Indian population [2].

Myocardial infarction can be defined from a number of different perspectives related to clinical, electrocardiographic (ECG), biochemical, and pathologic characteristics. It is accepted that the term myocardial infarction reflects death of cardiac myocytes caused by prolonged ischemia [4].

Coronary artery disease (CAD) is a leading cause of death throughout the world [5].The incidence of CAD has been halved in the west in past 3 years, but it has been doubled in the subcontinent. Moreover, people in our part of the world suffer from CAD at relatively younger age, i.e., about half of MI occurs under the age of 50 years [6]. The only way to get away with this problem is to evaluate the risk factors and try to modify them. The presence of major risk factors leads to advanced CAD in $2 \%$ of $15-19$ years old men and $0 \%$ in women. This figure increases to $20 \%$ and $8 \%$, respectively, in the presence of these risk factors in 30-34 years of age. These facts clearly show that early atherosclerosis is influenced by the risk factors for clinical CAD, so longterm prevention must begin in early adulthood [7].Very limited data are available regarding the prevalence of various risk factors for MI in our population. Keeping in view these facts, a study of risk factors in patients diagnosed with acute MI was planned, and the objective of this study was to determine the prevalence of risk factors which influence the increased rate of myocardial infarction in a tertiary care hospital, and this study helped to determine the recent trends in MI and the evolving risk factors for MI.

\section{METHODS}

We performed this prospective observational study with a sample size of 227. Data for this 6 months study were collected from patients admitted to a tertiary care hospital with myocardial infarction through patient interviews and case records. The inclusion criterion was any adult patient 60 years or older, of either sex, who was diagnosed with myocardial infarction. The variables collected in this study were age, sex, occupation, educational status, sleeping pattern, residential area, social habits including smoking alcoholism and physical activity, food habits, dairy and oil consumption, body mass index (BMI), family history, comorbid diseases, and drugs prescribed during hospital stay. Serial electrocardiograms and cardiac enzymes were also obtained in all patients. MI was defined by detection of rise in cardiac biomarkers of necrosis (cTn) with at least 1 value above the $99^{\text {th }}$ percentile upper reference limit, together with evidence of myocardial ischemia with at least 1 of the following: ECG changes indicative of new ischemia (new ST-T changes or new left bundle branch block), new pathological $\mathrm{Q}$ waves in at least 2 contiguous leads, imaging evidence of new loss 
of viable myocardium, or new wall motion abnormality. BMI was calculated as weight $(\mathrm{kg})$ divided by the square of height $\left(\mathrm{m}^{2}\right)$ and a patient with a value above 30 was categorized as obese. A data-base created in Microsoft Excel 2010 and the information was exported and analyzed. The protocol was approved by the institution ethical committee and the respective hospital authorities.

\section{RESULTS}

Data originating from 227 patients with a confirmed diagnosis of myocardial infarction were collected over 6-month period. The demographic characteristics of these patients are presented in Table 1.

Out of 227 patients, 184 patients $(80.64 \%)$ were not taking any exercise or physical activity, and 165 patients were presented with elevated lipid profile. Most of the patients were admitted with ST-elevation myocardial infarction (STEMI), and in that, only 55 patients were treated with fibrinolytics. Beta blockers are the most frequently used antihypertensive agents in myocardial infarction. Beta blockers reduce mortality during both acute and long-term management of myocardial infarction, and all the 227 patients were treated with beta blockers.

\section{DISCUSSION}

CAD remains a leading cause of death and exerts a heavy social and economical toll. Although mortality rates of CAD are declining overall in the affluent world, these rates have declined less for our population.

According to the results of this study, male sex is an important risk factor for MI. The predominant rate in men may relate to major risk factors such as smoking and sedentary lifestyle $[8,9]$. The lesser rate in women may also relate to the cardioprotective effect of estrogen. Estrogen receptors such as ER $\alpha, \mathrm{ER} \beta$, and GPR 30 confer cardioprotective effect against various stresses by preventing myocardial cell apoptosis and cardiac hypertrophy [10].

Patients above 20 years of age were included in this study. Most of the patients $(58.06 \%)$ were under the age group of 41-60 years (Table 2). The increased prevalence of CVD-related risk factors has been reported in this age group is due to lifestyle changes, work routines, culture influences, etc. [11].
Remaining $32.25 \%$ of patients were comes under the age group of 61-80 years. According to Shraddha et al., the increased prevalence in this age group can be collated to the demographic transition in India with a sharp decline in the death rate as well as the birth rate. The life expectancy of an average Indian male is 67.3 years and that of females is 69.6 years. This transition has brought a larger number of people to the age group where the CVD manifest [11]

Majority of hospital admissions with MI was from rural area, and the prevalence of MI in this population may be due to higher rates of smoking and diabetes. This population may also at higher risk of developing complications such as HF and cardiogenic shock [12].

Most of the patients admitted with MI were overweight and obese (approximately 75\%). So, obesity and overweight may contribute to the incidence of MI. Overweight and obesity increase the risk of AMI compared with normal individuals. The metabolic syndrome including abdominal obesity increased 2-fold in cardiovascular outcomes and 1.5 -fold in all-cause mortality. The increased mortality may due to impaired endothelium-dependent vasodilation through increased oxidative stress, leading to the reduced bioavailability of nitric oxide (NO). Endothelial dysfunction is an early finding in the arteriosclerosis and increases the incidence of CAD [13]

High-risk behaviors contributing to CVD are more common in individuals with low levels of education. We found that patient who did not attend school were at higher risk of acute myocardial infarction than those who did. Psychosocial behaviors associated with lack of education are likely to be responsible for this relation [14].

A total of 65 patients with sleep disorder, more women (60\%) than men $(40 \%)$ were reported difficulties in initiating and in maintaining sleep. A modestly positive association between short sleep duration as well as difficulty maintaining sleep and incident MI in women but not men was observed in many studies. Sleep may exert effects on the autonomic nervous system, systemic hemodynamics, cardiac function, endothelial function, and coagulation. Sleep disorders are associated with an increase in sympathetic activity, increases in blood pressure, or a decrease in glucose tolerance. Furthermore, recent research has demonstrated that sleep loss is accompanied by an elevation of inflammatory markers which could enhance the development of atherosclerosis [15].

Table 1: Demographic characteristic of study population

\begin{tabular}{|c|c|c|c|}
\hline Parameter & Category & Number of patients $(n=227)$ & Percentage of patients (\%) \\
\hline \multirow[t]{2}{*}{ Gender-wise distribution } & Male & 168 & 74.19 \\
\hline & Female & 59 & 25.81 \\
\hline \multirow[t]{4}{*}{ Age-wise distribution } & $21-40$ & 10 & 04.30 \\
\hline & $41-60$ & 132 & 58.06 \\
\hline & $61-80$ & 73 & 32.25 \\
\hline & Above 80 & 12 & 05.39 \\
\hline \multirow[t]{2}{*}{ Residential area-wise distribution } & Urban & 68 & 30.10 \\
\hline & Mixed & 30 & 12.92 \\
\hline \multirow[t]{4}{*}{ BMI characteristics } & Underweight & 0 & 0 \\
\hline & Normal & 59 & 25.80 \\
\hline & Overweight & 129 & 56.98 \\
\hline & Obese & 39 & 17.20 \\
\hline \multirow[t]{4}{*}{ Educational status } & Illiterate & 58 & 25.80 \\
\hline & Primary & 66 & 29.03 \\
\hline & Secondary & 46 & 20.43 \\
\hline & Degree & 23 & 09.67 \\
\hline \multirow[t]{7}{*}{ Occupational status } & Government worker & 25 & 11.01 \\
\hline & Business & 39 & 17.18 \\
\hline & Private workers & 42 & 18.50 \\
\hline & Farmer & 32 & 14.09 \\
\hline & Manual workers & 41 & 18.06 \\
\hline & Domestic & 30 & 13.21 \\
\hline & Nil & 18 & 07.92 \\
\hline
\end{tabular}


In this study population, the majority of them taking both vegetarian and non-vegetarian diet. According to Rao et al., non-vegetarian diet (Table 3) was associated with higher incidence of AMI, with a higher number of cases being meat eaters. Furthermore, the protective effect for vegetarians may be due to the fact that their diet is low in saturated fat and relatively high in unsaturated fats. Atherogenesis and thrombogenesis are also believed to be lower in vegetarians as their platelet phospholipids linoleate, and antioxidant concentrations are higher, which is likely to lead reduced oxidation of low-density lipoproteins (LDL) [16].

There is no association for coffee and tea consumption with the risk of myocardial infarction, and there is an inverse association between tea and coronary heart disease death. The two possible explanations for the inverse association between tea and the reduced risk of myocardial infarction is that flavonoids in black tea may reduce myocardial infarction by inhibiting low-density lipoprotein cholesterol oxidation, reducing platelet aggregation, or reducing ischemic damage and increased tea consumption has been associated with little or no improvement in lipid profiles [17]. There is no relationship between dairy consumption and risk of myocardial infarction or change in serum lipid level [18].

Both dietary and adipose levels of $\alpha$-linolenic acid (which is present in all oils of plant origin like sunflower oil) were associated with a large and significant reduction in the risk of non-fatal acute MI. $\alpha$-Linolenic acid could exert direct protective anti-inflammatory effects. It has been shown that $\alpha$-linolenic acid decreases the nuclear transcription factor $\mathrm{KB}$, a major transcription factor that is involved in the regulation of inflammatory genes. Furthermore, $\alpha$-linolenic acid inhibits the production of NO and downregulates inducible NO synthase cyclooxygenase-2, and tumor necrosis factor- $\mathrm{R}$ gene expression in macrophages [19].

According to National Heart, Lung, and Blood Institute (NHLBI) Family Heart Study, individuals who reported $\sim 0.6 \mathrm{~g} / \mathrm{d}$ higher linolenic acid intake had lower blood pressure, lower plasma triglycerides concentration, fewer calcified atherosclerosis plaques, and less carotid

Table 2: Sleeping status of study population

\begin{tabular}{llll}
\hline Parameter & Category & $\begin{array}{l}\text { Number of patients } \\
(\mathbf{n = 2 2 7})\end{array}$ & $\begin{array}{l}\text { Percentage } \\
\text { (\%) }\end{array}$ \\
\hline Sleeping status & Normal & 162 & 71.36 \\
& Sleep disorder & 65 & 28.64 \\
\hline
\end{tabular}

Table 3: Food habits of study population

\begin{tabular}{llll}
\hline Parameter & Category & $\begin{array}{l}\text { Number of } \\
\text { patients (n=227) }\end{array}$ & $\begin{array}{l}\text { Percentage } \\
\text { (\%) }\end{array}$ \\
\hline Diet pattern & Non-vegetarian & 27 & 11.82 \\
& Vegetarian & 22 & 09.67 \\
\multirow{2}{*}{ Dairy consumption } & Mixed & 178 & 78.51 \\
& Milk & 66 & 29.04 \\
Oil consumption & Coffee and tea & 161 & 70.96 \\
& Coconut oil & 66 & 29.03 \\
& Sun flower oil & 132 & 58.06 \\
& Ground nut oil & 07 & 03.22 \\
& Palm oil & 22 & 09.69 \\
\hline
\end{tabular}

Table 4: Social habits of study population

\begin{tabular}{llll}
\hline Parameter & Category & $\begin{array}{l}\text { Number of patients } \\
(\mathbf{n = 2 2 7})\end{array}$ & Percentage (\%) \\
\hline Social habits & Smokers & 149 & 65.63 \\
& Alcoholics & 144 & 63.43 \\
& Betel nut users & 142 & 62.28 \\
\hline
\end{tabular}

atherosclerosis. Coconut oil cannot cause atherosclerosis and CVD, because according to the universally accepted lipid-heart theory, high saturated fats cause hypercholesterolemia and CVD, coconut oil consists predominantly of $65 \%$ medium-chain fatty acids (MCFAs), and MCFAs are metabolized rapidly in the liver to energy and do not participate in the biosynthesis and transport of harmful cholesterol. Coconut oil, in fact, tends to raise the high-density lipoprotein (HDL) and lower the LDL: HDL ratio. Coconut oil is not deposited in adipose tissues, and therefore, does not lead to obesity. It is primarily an energy supplier and as fast a supplier of energy as sugar [20]. So, the consumption of sunflower or coconut oil has no effect on increased incidence of MI.

Saturated fat intake has been related to acute myocardial infarction in adults. Ghee contains cholesterol oxides that are thought to be related to atherosclerosis. In adipose tissue of patients who use ghee for cooking compared with other types of non-hydrogenated oils, a higher percentage of trans fatty acids, known to increase the risk of CVD. Although previous studies in South Asian populations indicate a high consumption of ghee in India, data for an independent relation between ghee and CVD in the same population are conflicting. Our findings suggest that ghee consumption is a potential target for preventive strategies [14].

Exercise or physical activity can reduce or prevent CVD, and physical inactivity may contribute to CVD. In this study, most of the patients were not taking any exercise or physical activity. This study is similar to the study of Thompson et al. and it states that physical activity both prevents and helps treat many established atherosclerotic risk factors, including elevated blood pressure, elevated triglyceride concentrations, low HDL cholesterol (HDL-C) concentrations, insulin resistance and glucose intolerance, and obesity. Exercise in combination with weight loss can decrease LDL cholesterol (LDL-C) concentrations and limit the reduction in HDL-C that often occurs with a reduction in dietary saturated fat [21].

In our study, most of the patients were beedi smokers than cigarette smokers and beedis (unfiltered cigarettes) which deliver a higher content of $\mathrm{CO}$ and nicotine than conventional Indian cigarettes (Table 4). AMI was almost 4-fold higher for current smokers compared to non-smokers. Smoking acts synergistically with other risk factors substantially increasing the risk of CVD. Smoking is associated with endothelial dysfunction and can precipitate coronary spasm which may lead to CAD. Hanartty et al. also made the same results as like this study $[8,22,23]$

Heavy drinking (taken as more than $180 \mathrm{ml} /$ day) to be a salient risk factor in the incidence of AMI and moderate drinkers were found to be the lowest risk category. This can be explained by a polymorphism in the gene for alcohol dehydrogenase type 3 (ADH3) alters the rate of alcohol metabolism, and hence, moderate drinkers who are homozygous for the slow-oxidizing ADH3 allele have higher HDL levels and substantially decreased the risk of MI [24].

Maria et al. studied the relationship between family history and AMI, and they found that family history of acute myocardial infarction was a strong and independent risk factor for myocardial infarction. This study was also made the same results. The antecedent of $\geq 1$ first-degree relative with acute myocardial infarction increased the risk about 2 -fold in men and nearly 3 -fold in woman, with a significant trend in risk in the number of relative's affected [25].

Diabetes is an independent risk factor for $\mathrm{MI}$; it is considered to be equivalent to CAD. In this study, $73 \%$ of patients had diabetes (Table 5). Cardiac autonomic neuropathy may be present in nearly $50 \%$ of the diabetic population with CAD and can cause both diastolic and systolic dysfunction. The relation between MI and Hypertension is closely correlated with salt intake, alcohol intake, and obesity. The mean salt intake among Indians is $8.5 \mathrm{~g} /$ day, which is higher than the recommended level. Dyslipidemia in association with one or other 
Table 5: Comorbid diseases and family history of study population

\begin{tabular}{|c|c|c|c|}
\hline Parameter & Category & Number of patients $(n=227)$ & Percentage $(\%)$ \\
\hline \multirow[t]{8}{*}{ Comorbid diseases } & Hypertension alone & 23 & 16.12 \\
\hline & Diabetes mellitus alone & 31 & 18.27 \\
\hline & Dyslipidemia (DL) alone & 26 & 11.82 \\
\hline & CAD & 31 & 16.12 \\
\hline & $\mathrm{HTN}+\mathrm{DM}$ & 24 & 11.82 \\
\hline & $\mathrm{HTN}+\mathrm{DL}$ & 15 & 08.60 \\
\hline & $\mathrm{DM}+\mathrm{DL}$ & 09 & 06.45 \\
\hline & Without comorbidities & 50 & 22.02 \\
\hline \multirow[t]{6}{*}{ Family history } & Father & 44 & 19.35 \\
\hline & Mother & 34 & 15.05 \\
\hline & Father and mother & 49 & 21.50 \\
\hline & Brother and sister & 24 & 10.75 \\
\hline & Others (grandfather, grandmother, etc.) & 07 & 03.22 \\
\hline & Nil & 69 & 30.10 \\
\hline
\end{tabular}

CAD: Coronary artery disease, DM: Diabetes mellitus

Table 6: Drug prescribing pattern in ST elevated MI

\begin{tabular}{|c|c|c|c|c|}
\hline \multirow[t]{2}{*}{ Drugs prescribed } & \multicolumn{3}{|c|}{$\begin{array}{l}\text { Number of patients } \\
(n=105)\end{array}$} & \multirow[t]{2}{*}{$\begin{array}{l}\text { Percentage } \\
(\%)\end{array}$} \\
\hline & Male & Female & Total & \\
\hline Fibrinolytics & 48 & 07 & 55 & 52.23 \\
\hline \multicolumn{5}{|l|}{ Anti platelets } \\
\hline Aspirin & 90 & 15 & 105 & 100 \\
\hline Clopidogrel & 90 & 15 & 105 & 100 \\
\hline \multicolumn{5}{|l|}{ Anticoagulants } \\
\hline Enoxaparin & 76 & 12 & 88 & 83.01 \\
\hline Warfarin & 08 & 03 & 11 & 11.32 \\
\hline Heparin & 06 & 00 & 06 & 05.66 \\
\hline \multicolumn{5}{|l|}{ Beta blocker } \\
\hline Bisoprolol & 90 & 15 & 105 & 100 \\
\hline \multicolumn{5}{|l|}{ Ace inhibitors } \\
\hline Enalapril & 18 & 03 & 21 & 20.75 \\
\hline \multicolumn{5}{|l|}{ Angiotensin receptor blocker } \\
\hline Losartan & 10 & 02 & 12 & 11.32 \\
\hline \multicolumn{5}{|l|}{ Statins } \\
\hline Atorvastatin & 86 & 15 & 101 & 96.22 \\
\hline Rosuvastatin & 04 & 00 & 04 & 03.78 \\
\hline \multicolumn{5}{|l|}{ Calcium channel blockers } \\
\hline Amlodipine & 12 & 02 & 14 & 13.33 \\
\hline Nitrates pain management & 82 & 12 & 94 & 89.52 \\
\hline Opioid analgesics & 08 & 02 & 10 & 09.43 \\
\hline NSAIDS & 47 & 05 & 52 & 49.05 \\
\hline Diazepam & 27 & 05 & 32 & 30.18 \\
\hline NSAIDS+Diazepam & 08 & 03 & 11 & 11.32 \\
\hline
\end{tabular}

risk factors contributed significantly to MI. Hypertension and diabetes together result in more cardiac fibrosis than when either occurs alone. Endothelial dysfunction may impair coronary perfusion at the microvascular level, resulting in ischemia [26].

The drugs were prescribed according to 2014 ACCF/AHA Guideline for the management of STEMI and standard treatment guideline for myocardial infarction by Government of Tamil Nadu. Out of 105 patients, 55 were treated with fibrinolytic therapy. 50 patients were excluded from this therapy (Table 6). The exclusion may due to:

- Any previous history of hemorrhagic stroke

- History of stroke, dementia, or central nervous system damage within 1 year

- Head trauma or brain surgery within 6 months

- Known intracranial neoplasm

- $\quad$ Suspected aortic dissection

- Internal bleeding within 6 weeks

- Active bleeding or known bleeding disorder

- Major surgery, trauma, or bleeding within, 6 weeks

- Traumatic cardiopulmonary resuscitation within 3 weeks
- $\quad$ Or may due to delayed admission to hospital [27].

Beta blockers are the most frequently used antihypertensive agents in myocardial infarction. Beta blockers reduce mortality during both acute and long-term management of myocardial infarction. Administration of intravenous beta blockers within 12-24 hrs of infarction, followed by oral therapy, has been found to reduce the mortality rate approximately $13 \%$ within the first week of infarction. This study indicated that the most marked reduction (25\%) occurs in the first 2 days after infarction [28].

\section{CONCLUSION}

Myocardial infarction is the fatal cause of death more common in late middle-aged males and smoking is the major risk factor. Fasting hyperglycemia, hypertension, central obesity, higher socioeconomic status, increased levels of triglycerides, and current smoking are major risk factors for the first episode of AMI in our population. Smoking is responsible for a higher proportion of acute myocardial infarction cases in young men, while the lack of regular leisure-time physical activity practice has a higher impact among the older. The clustering of risk factors particularly three or more risk factors in an individual predispose to MI at relatively younger age. If a young male who is a smoker or a young female who is diabetic, presents in the emergency room with chest pain, always suspect CAD. Other conventional risk factors are also prevalent in our population, but oil consumption and dairy consumption are not a major health problem for us. Beta blockers should be administered to all post-MI patients without a contraindication and should be continued indefinitely, as recommended in the recent 2014 AHA/ACC guidelines. To increase awareness regarding moderate physical activity, cessation of cigarette smoking, increased daily consumption of fruits and vegetables, and also identification of new and less known factors to tackle the problem and reduce the prevalence of AMI.

\section{REFERENCES}

1. Yusuf S, Reddy S, Ounpuu S, Anand S. Global burden of cardiovascular diseases: Part I: General considerations, the epidemiologic transition, risk factors, and impact of urbanization. Circulation 2001;104(22):2746-53.

2. Yusuf S, Hawken S, Ounpuu S, Dans T, Avezum A, Lanas F, et al. Effect of potentially modifible risk factors associated with myocardial infarction in 52 countries (the INTERHEART study): Case-control study. Lancet 2004;364(9438):937-52.

3. Gupta R, Joshi P, Mohan V, Reddy KS, Yusuf S. Epidemiology and causation of coronary heart disease and stroke in India. Heart 2008;94(1):16-26.

4. The Joint European Society of Cardiology/American College of Cardiology Committee. Myocardial infarction redefined-A consensus document of the joint European Society of Cardiology/American College of Cardiology for the redefinition of myocardial infarction. Eur 
Heart J 2000;21:1502-13

5. Murray CJ, Lopez AD. Mortality by cause for eight regions of the world: Global burden of disease study. Lancet 1997;349(9061):1269-76.

6. Enas EA, Senthilkumar A. Coronary artery disease in Asian Indians: An update and review. Int J Cardiol 2002;1(2):1-34.

7. McGill HC Jr, McMahan CA, Zieske AW, Tracy RE, Malcom GT, Herderick EE, et al. Association of coronary heart disease risk factors with microscopic qualities of coronary atherosclerosis in youth Circulation 2000;102(4):374-9.

8. Tanna NA, Srivastava RS, Tanna VA, Vaishnani HV. The role of unknown risk factors in myocardial infarction. Int J Biomed Adv Res 2013;4(6):6-10.

9. Hanratty B, Lawlor DA, Robinson MB, Sapsford RJ, Greenwood D, Hall A. Sex differences in risk factors, treatment and mortality after acute myocardial infarction: An observational study. J Epidemiol Community Health 2000;54(12):912-6.

10. Ding YL, Fuu JT, Chang HT, Chih YH. Mechanisms governing the protective effect of $17 \mathrm{~b}$-estradiol and estrogen receptors against cardiomyocyte injury. Bio Med 2011;4:21-8.

11. Shraddha C, Bani TA. Prevalence of cardiovascular disease in India and it is economic impact - A review. Int J Sci Res Publ 2013;3(10):10-7.

12. Bergström G, Redfors B, Angerås O, Dworeck C, Shao Y, Haraldsson I, et al. Low socioeconomic status of a patient's residential area is associated with worse prognosis after acute myocardial infarction in Sweden. Int J Cardiol 2015;182:141-7.

13. Mottillo S, Filion KB, Genest J, Joseph L, Pilote L, Poirier P, et al. The metabolic syndrome and cardiovascular risk a systematic review and meta-analysis. J Am Coll Cardiol 2010;56(14):1113-32.

14. Ismail J, Jafar TH, Jafary FH, White F, Faruqui AM, Chaturvedi N. Risk factors for non-fatal myocardial infarction in young South Asian adults. Heart 2004;90(3):259-63.

15. Meisinger C, Heier M, Löwel H, Schneider A, Döring A. Sleep duration and sleep complaints and risk of myocardial infarction in middle-aged men and women from the general population: The MONICA/KORA Augsburg cohort study. Sleep 2007;30(9):1121-7.

16. Rao V, Rao P, Carvalho N. Risk factors for acute myocardial infarction in Coastal region of India: A case control study. Heart India 2014;2(3):70-5.

17. Howard DS, Michael GJ, Julie EB, Charles HH. Coffee and tea intake and the risk of myocardial infarction. Am $\mathrm{J}$ Epidemiol 1991;149(2):162-7.

18. Amir M. Association between consumption of dairy products and serum lipids in subjects with coronary heart disease. Public Health Nutr 2013;1:1-39.

19. Campos H, Baylin A, Willett WC. Alpha-linolenic acid and risk of nonfatal acute myocardial infarction. Circulation 2008;118(4):339-45.

20. Conrado SD. Coconut oil: Atherogenic or not? Philipp J Cardiol 2003;31(3):97-104

21. Thompson DP, David B, Pina L, Gary JB, Mark AW, Bess HM, et al. Exercise and physical activity in the prevention and treatment of atherosclerotic cardiovascular disease. Circulation 2003;107(24):3109-16.

22. Faisal AW, Ayub M, Waseem T, Khan RS, Hasnain SS. Risk factors in young patients of acute myocardial infarction. J Ayub Med Coll Abbottabad 2011;23(3):10-3.

23. Ghosh JD, Dasgupta S, Rajni, Banerjee I, Biswas UK. Risk factor for acute myocardial infarction among rural population of Bihar: A community based study. Sch J Appl Med Sci 2014;2(3C):1075-80.

24. Vinay R, Prasannalakshmi R, Nikita C. Risk factors for acute myocardia infarction in Coastal region of India: A case-control study. Heart India 2014;2(3):70-5

25. Roncaglioni MC, Santoro L, D’Avanzo B, Negri E, Nobili A, Ledda A et al. Role of family history in patients with myocardial infarction. An Italian case-control study. GISSI-EFRIM investigators. Circulation 1992;85(6):2065-72.

26. Richard WN, Stuart Z. Acute myocardial infarction in diabetes mellitus Circulation 1998;97(23):12-5

27. Joseph DP, Robert LT, Gary CY, Gary RM. Pharmacotherapy. A Pathophyphysiologic Approach. $7^{\text {th }}$ ed. United States of America. M. Graw Hill; 2008.

28. Howard PA, Ellerbeck EF. Optimizing beta-blocker use after myocardial infarction. Am Fam Physician 2000;62(8):1853-60, 1865-6. 